\title{
Point mass perturbations of spectral measures
}

\author{
Rafael del Rio* \\ Departamento de Física Matemática \\ Instituto de Investigaciones en Matemáticas Aplicadas y en Sistemas \\ Universidad Nacional Autónoma de México \\ C.P. 04510, Ciudad de México \\ delrio@iimas.unam.mx \\ Luis O. Silva ${ }^{\dagger}$ \\ Departamento de Física Matemática \\ Instituto de Investigaciones en Matemáticas Aplicadas y en Sistemas \\ Universidad Nacional Autónoma de México \\ C.P. 04510, Ciudad de México \\ silva@iimas.unam.mx

\section{Julio H. Toloza} \\ INMABB \\ Departamento de Matemática \\ Universidad Nacional del Sur (UNS) - CONICET \\ Bahía Blanca, Argentina \\ julio.toloza@uns.edu.ar
}

\begin{abstract}
Using a generalization of the moment problem and the extremal properties of spectral measures corresponding to the selfadjoint extensions of a regular symmetric operator, we study point mass perturbations of spectral measures. We obtain general results for a wide class of operators and apply them to the analysis of point mass perturbations of spectral measures pertaining to Bessel and generalized Schrödinger operators.
\end{abstract}

Mathematics Subject Classification(2010): 47B32, 34L05, 47B36

Keywords: spectral measure; de Branges spaces; perturbations of measures

*Supported by UNAM-DGAPA-PAPIIT IN110818

†Supported by UNAM-DGAPA-PAPIIT IN110818 and SEP-CONACYT CB-2015 254062

${ }^{\ddagger}$ Partially supported by CONICET (Argentina) under grant PIP $11220150100327 \mathrm{CO}$ 


\section{Introduction}

The motivation of this work stems from the intriguing fact that very small perturbations of a measure may destroy the density of polynomials in the space of square integrable functions with respect to that measure. More precisely: one can find a Borel measure $\mu$ such that the polynomials are dense in $L_{2}(\mathbb{R}, \mu)$, but they are no longer dense in $L_{2}(\mathbb{R}, \widetilde{\mu})$ when $\widetilde{\mu}$ is obtained by adding a point mass to $\mu$, that is

$$
\widetilde{\mu}=\mu+\delta_{\lambda}, \quad \lambda \notin \operatorname{supp} \mu,
$$

where $\delta_{\lambda}$ denotes a discrete measure which gives weight only to the point $\lambda$. This instability phenomenon pertains to the classical Hamburger moment problem and a particular realization corresponds to the following reformulation. If $\mu$ is an $N$-extremal solution to an indeterminate moment problem, then $\widetilde{\mu}$ is a non- $N$ extremal solution to a moment problem (see [1, Thm. 2.3.2] and [10, Prop. 4.1]). Remarkably, the theory of the Hamburger moments is the theory of Jacobi operators, i.e. operators in $l_{2}(\mathbb{N})$ generated by semi-infinite tridiagonal symmetric matrices (see Example 6.1). In this context, the mentioned phenomenon is paraphrased as follows: if $\mu$ is the spectral measure of a canonical selfadjoint extension of a nonselfadjoint Jacobi operator, i. e. a selfadjoint restriction of its adjoint (see Section 2.1), then there is no canonical selfadjoint extension of any Jacobi operator having $\widetilde{\mu}$ as its spectral measure.

This paper is devoted to studying the situation illustrated above in a setting that allows us to consider not only nonselfadjoint Jacobi operators, but a wide class of regular symmetric operators with deficiency indices $(1,1)$, including regular and certain singular Schrödinger operators. Our approach is based on Krein's theory of symmetric operators [20-22], the theory of generalized extensions due to Nalmark [32] and a generalization of the moment problem originally proposed by Livsič [28]. This theoretical scaffold allows us to prove several results on point mass perturbations of spectral measures presented in Section 5. The central theorem (Theorem 5.4) is general enough to be applied to the analysis of point mass perturbation of spectral measures related to generalized Schrödinger operators with measures and Bessel operators. This analysis led to Theorems 6.2, 6.4, and 6.5.

The spectrum of a regular Schrödinger operator defined by any selfadjoint boundary conditions has the following asymptotic behavior

$$
\lambda_{n}=c n^{2}+O(1), \quad \text { as } n \rightarrow \infty,
$$

(see [33]). From this formula, by a straightforward argument (see Remark 6.3), one concludes that if $\mu$ is the spectral measure of a regular Schrödinger operator given by selfadjoint boundary conditions, then $\widetilde{\mu}$, defined as in (1.1), cannot be 
the spectral measure of any regular Schrödinger operator with any selfadjoint boundary conditions no matter which potential function is considered. It is worth remarking that the results of Section 5 provide a way for dealing with point mass perturbation of spectral measures for a wide range of operators even in the case when asymptotics of the kind of (1.2) are not available. This is illustrated in Examples 6.2 and 6.3.

Let us elaborate on the theoretical framework developed in this paper which is of interest in itself irrespective of the results given in Sections 4 and 6. The classical theory of moments tells us that to account for all solutions to the moment problem, one has to recur to Naimmark's theory of generalized extensions [31,32] since the solutions are the generalized spectral measures (see Definition 2.5) of the corresponding Jacobi operator. Here we remark that, despite the classicality of the theory, relatively recent developments $[7,45]$ make use of [32] to construct solutions to the classical moment problem with the measure having certain predefined properties. Our approach makes use not only of Naŭmark's theory, but also Krein's theory of symmetric operators with deficiency indices $(1,1)$ [20-22] to study the set of measures related to an arbitrary regular symmetric operator not necessarily entire [15]. This allows us to characterize the spectral measures associated with the operator (Theorem 3.4) and describe the extremal properties of measures pertaining to this operator's selfadjoint extensions (Theorem 3.6). The next step in our method is the use of the functional model for regular symmetric operators $[38,39]$ to map any regular symmetric operator into the multiplication operator in a de Branges spaces. This reformulation leads us directly to the generalized moment problem.

There are various ways of generalizing the moment problem. Here, we have in mind a proposal of generalization that goes back to Livsič [28] for whom the moment problem consists in finding a measure $\rho$ such that the inner product in a Hilbert space of functions is expressed through the inner product in $L_{2}(\mathbb{R}, \rho)$. A related setting of the generalized moment problem is found in the introduction of [44]. As pointed out in [36, Chap. 6], de Branges treated this problem using a different terminology when the Hilbert space of functions is a de Branges space [9]. In this work, we also consider the generalization of [28] with the underlying Hilbert space being a de Branges space, but in contrast to [9] and [36, Chap. 6], we focus on the extremal properties of the solutions to the generalized moment problem.

Let us outline the material of this paper. Section 2 presents classical results that are included for the sake of completeness and to introduce the notation. Section 3 study the extremal properties of measures associated with regular symmetric operators. A short survey on de Branges spaces theory and the generalized moment problem are the subject of Section 4. In this section, we also present the functional model for regular symmetric operators by means of which we connect the results on de Branges spaces to our class of operators. In Sec- 
tion 5, we prove the main results related to perturbation of spectral measures that results when adding a mass at a single point. Here we also touch upon the fact that certain functions which are dense in $L_{2}(\mathbb{R}, \mu)$ are no longer dense in $L_{2}(\mathbb{R}, \mu)$ (see $(1.1)$ ). These results may have several applications to the inverse spectral analysis of operators as is shown in [10] for Jacobi operators. Finally, in Section 6, we illustrate the results of this work. The first example is used to put our results in the context of the classical moment problem. The other two examples present nontrivial results pertaining differential operators which include regular and singular Schrödinger operators.

\section{Preliminaries}

Let $\mathcal{H}$ be a separable Hilbert space. The inner product in it is denoted by $\langle\cdot, \cdot\rangle$ where we agree that the left-hand-side argument is anti-linear. We denote by $\oplus, \ominus$, and $\dot{+}$ the orthogonal sum, the orthogonal complement, and the direct sum of linear spaces, respectively.

Let $A$ be a not necessarily densely defined linear closed symmetric operator. If $A$ is not densely defined, then $A^{*}$ is a multivalued linear operator (linear relation). In this case, the symmetry condition, $A \subset A^{*}$, remains valid (as subspaces of $\mathcal{H} \oplus \mathcal{H})$. The deficiency indices $n_{+}(A)$ and $n_{-}(A)$ of $A$ are defined as the dimension of $\mathcal{H} \ominus \operatorname{ran}(A-z I)$, when $z \in \mathbb{C}_{+}:=\{z \in \mathbb{C}: \operatorname{Im} z>0\}$ and $z \in \mathbb{C}_{-}:=\{z \in \mathbb{C}: \operatorname{Im} z<0\}$, respectively. If $A$ has equal deficiency indices, then it has canonical selfadjoint extensions (that is, selfadjoint restrictions of $\left.A^{*}\right)$, with some of these extensions being proper linear relations if $\operatorname{dom}(A)$ is not dense in $\mathcal{H}$ [16, Prop. 5.4].

What follows is a brief revision of Naı̆mark's theory of selfadjoint extensions to a larger space [31,32] (see also [2, Appendix 1] and [44]), as well as some results from Krein's theory on regular symmetric operators [15, 22].

\subsection{Generalized resolvents}

Definition 2.1. Consider a closed symmetric operator $A$. Let $A^{+}$be a selfadjoint extension of $A$ in a Hilbert space $\mathcal{H}^{+} \supset \mathcal{H}\left(\mathcal{H}^{+}=\mathcal{H}\right.$ is allowed $)$. For $w \in \mathbb{C}$ and $z \in \mathbb{C} \backslash \operatorname{spec}\left(A^{+}\right)$, define

$$
V_{A^{+}}(w, z):=\left(A^{+}-w I\right)\left(A^{+}-z I\right)^{-1}=I+(z-w) R^{+}(z),
$$

where $R^{+}$is the resolvent of $A^{+}$(cf. [15, Ch. 1 Sec. 2.1]).

From the first resolvent identity (see [6, Ch. 3 Sec. 7]), one verifies that

$$
V_{A^{+}}(w, z)=V_{A^{+}}(z, w)^{-1} \text { and } V_{A^{+}}(w, z) V_{A^{+}}(z, v)=V_{A^{+}}(w, v)
$$


hold true for any $v, w, z \in \mathbb{C} \backslash \operatorname{spec}\left(A^{+}\right)$. Also, it is straightforward to establish that

$$
V_{A^{+}}(w, z)^{*}=V_{A^{+}}(\bar{w}, \bar{z}) .
$$

The following assertion arises by combining the first identity in (2.2) with (2.3) (see [15, Ch. 1 Sec. 2.1]).

Proposition 2.2. Let $z \in \mathbb{C}$ and $w \in \mathbb{C}_{+}$. For any selfadjoint extension $A^{+}$ of $A$ such that $z \notin \operatorname{spec}\left(A^{+}\right)$, the operator $V_{A^{+}}(w, z)$ maps $\mathcal{H}^{+} \ominus \operatorname{ran}(A-\bar{w} I)$ injectively onto $\mathcal{H}^{+} \ominus \operatorname{ran}(A-\bar{z} I)$.

Definition 2.3. A scalar function with domain of analyticity containing $\mathbb{C}_{+}$is a Herglotz function whenever $\operatorname{Im} z>0 \Longrightarrow \operatorname{Im} f(z) \geq 0$. By this definition, any real constant is a Herglotz function. Denote by $\mathfrak{R}$ the union of the class of Herglotz functions and the constant $\infty$.

Definition 2.4. Denote by $P^{+}$the orthogonal projector of $\mathcal{H}^{+}$onto $\mathcal{H}$ and by $R^{+}(z)$ the resolvent of $A^{+}$. The operator

$$
R(z):=P^{+} R^{+}(z) \uparrow_{\mathcal{H}}, \quad z \in \mathbb{C} \backslash \mathbb{R}
$$

is a generalized resolvent of $A$ [32] (see also [2, Appendix 1] and [44, § 3]).

Fix a canonical selfadjoint extension of a closed symmetric operator $A$ with deficiency indices $n_{+}(A)=n_{-}(A)=1$ and denote it by $A_{\infty}$. For $w \in \mathbb{C}_{+}$and $z \in \mathbb{C} \backslash \operatorname{spec}\left(A_{\infty}\right)$, define

$$
\psi(z):=V_{A_{\infty}}(w, z) \psi
$$

where $\psi \in \operatorname{ker}\left(A^{*}-w I\right) \backslash\{0\}$ (cf. [2, Appendix 1, Sec. 4, Eq. 2']).

Let $R_{\infty}(z)$ be resolvent of $A_{\infty}$. Given $\tau \in \mathfrak{R}$, define

$$
R_{\tau}(z):=R_{\infty}(z)-\frac{\langle\psi(\bar{z}), \cdot\rangle \psi(z)}{\tau(z)+Q(z)}
$$

where

$$
Q(z):=i \operatorname{Im} w+(z-w)\langle\psi(\bar{z}), \psi(w)\rangle
$$

is the so-called $Q$-function (see [2, Appendix 1 Eq. 8]). $R_{\tau}(z)$ is the resolvent of a canonical selfadjoint extension of $A$ if and only if $\tau(z) \equiv c \in \mathbb{R} \cup\{\infty\}$. Moreover, once a canonical selfadjoint extension $A_{\infty}$ is fixed, (2.5) induces a bijection between $\mathfrak{R}$ and the set of all generalized resolvents of $A$ (see [11, Thm. 6.2], also $[19,23,24,32,43])$. Equation $(2.5)$ is known as the Krein-Naĭmark formula.

Definition 2.5. Let $E_{A^{+}}$denote the spectral family of $A^{+}$given by the spectral theorem. Let $\mathfrak{X}$ denote the $\sigma$-algebra of Borel sets on $\mathbb{R}$. Given a selfadjoint 
extension $A^{+}$of a symmetric operator $A$, the map

$$
F_{A^{+}}(\partial):=P^{+} E_{A^{+}}(\partial) \Upsilon_{\mathcal{H}}, \quad \partial \in \mathfrak{X},
$$

is called a generalized spectral family of the operator $A$. Furthermore, given $\phi \in \mathcal{H} \backslash\{0\}$, we define

$$
\sigma_{A^{+}, \phi}(\partial):=\left\langle\phi, F_{A^{+}}(\partial) \phi\right\rangle_{\mathcal{H}}, \quad \partial \in \mathfrak{X}
$$

We call $\sigma_{A^{+}, \phi}$ the generalized spectral measure of $A$ associated with $\phi$ and $A^{+}$.

Note that

$$
\sigma_{A^{+}, \phi}(\partial)=\left\langle\phi, E_{A^{+}}(\partial) \phi\right\rangle_{\mathcal{H}^{+}}, \quad \partial \in \mathfrak{X},
$$

so $\sigma_{A^{+}, \phi}$ is also the spectral measure of the selfadjoint extension $A^{+}$associated with $\phi \in \mathcal{H}$ and therefore it is a nonnegative finite scalar measure. On the basis of [2, Appendix I Thm. 2], the next result holds true.

Proposition 2.6. $F$ is a generalized spectral family of $A$ (that is, $F=F_{A^{+}}$for some $\left.A^{+}\right)$if and only if the operator-valued function $F(t):=F(-\infty, t)(t \in \mathbb{R})$ satisfies, for any $\phi \in \mathcal{H}$, the following conditions:

(i) The function $\langle\phi, F(t) \phi\rangle$ does not decrease when $t$ increases.

(ii) $F(t) \phi$ is left continuous.

(iii) $F(t) \phi \underset{t \rightarrow-\infty}{\longrightarrow} 0$ and $F(t) \phi \underset{t \rightarrow \infty}{\longrightarrow} \phi$.

(iv) If $\phi \in \operatorname{dom}(A)$, then

$$
\|A \phi\|^{2}=\int_{\mathbb{R}} t^{2} d\langle\phi, F(t) \phi\rangle \quad \text { and } \quad A \phi=\int_{\mathbb{R}} t d F(t) \phi .
$$

The next statement is proven on the basis of Proposition 2.6 (see [2, Appendix 1, pp. 141-143]).

Proposition 2.7. There is a bijection between $\mathfrak{R}$ and the generalized spectral family of any symmetric operator with deficiency indices $(1,1)$. The bijection is given by the combination of (2.5) and the identity

$$
\langle\phi, R(z) \phi\rangle_{\mathcal{H}}=\left\langle\phi, R^{+}(z) \phi\right\rangle_{\mathcal{H}^{+}}=\int_{\mathbb{R}} \frac{d \sigma_{A^{+}, \phi}(t)}{t-z} .
$$

Moreover, the same bijection gives a one-to-one map between $\mathbb{R} \cup\{\infty\}$ and the spectral family corresponding to canonical selfadjoint extensions of $A$. 


\subsection{Regular symmetric operators and gauges}

Definition 2.8. A closed symmetric nonselfadjoint operator is said to be completely nonselfadjoint if it is not a nontrivial orthogonal sum of a symmetric and a selfadjoint operators.

Definition 2.9. The complex number $z$ is in the set of points of regular type of a closed operator $T$ when there exists $c_{z}>0$ such that

$$
\|(T-z I) \phi\| \geq c_{z}\|\phi\| \text { for all } \phi \in \operatorname{dom}(T) .
$$

The complement of the set of points of regular type of $T$ is called the spectral kernel of $T$.

Definition 2.10. A closed operator $T$ is regular when the set of points of regular type is the whole complex plane.

A regular, closed symmetric operator is necessarily completely nonselfadjoint since regularity implies that the spectral kernel is empty and, therefore, the operator cannot have selfadjoint parts. On the other hand, there are many completely nonselfadjoint operators that are not regular $[41$, Sec. 2]. We note that the dimension of $\operatorname{ker}\left(A^{*}-z I\right)$ is constant for all $z \in \mathbb{C}$ whenever $A$ is regular.

Definition 2.11. Let $\mathcal{S}(\mathcal{H})$ denote the set of regular, closed symmetric operators in $\mathcal{H}$ with deficiency indices $n_{+}(A)=n_{-}(A)=1$.

The next assertion is well known for densely defined symmetric operators $[3,12,15]$. A proof for the case under discussion in this work can be found in [39, Prop. 2.4].

Proposition 2.12. Let $A$ be in $\mathcal{S}(\mathcal{H})$. The following statements are true:

(i) The spectrum of every canonical selfadjoint extension of A consists solely of isolated eigenvalues of multiplicity one.

(ii) Every real number is part of the spectrum of one, and only one, canonical selfadjoint extension of $A$.

(iii) The spectra of the canonical selfadjoint extensions of $A$ are pairwise interlaced.

Note that item (i) above means that every selfadjoint extension of $A$ is a simple operator [2, Sec. 69]. Also, item (iii) above (together with the fact that under extensions the spectral kernel does not decrease) implies that $A$ is regular if and only if the spectra of any two selfadjoint extensions of $A$ do not intersect. 
For any $A \in \mathcal{S}(\mathcal{H})$, let $\kappa \in \mathcal{H}$ be such that

$$
\mathcal{H}=\operatorname{ran}\left(A-z_{0} I\right) \dot{+} \operatorname{span}\{\kappa\},
$$

for some $z_{0} \in \mathbb{C}$. For this vector $\kappa$, consider the set

$$
\{z \in \mathbb{C}: \kappa \in \operatorname{ran}(A-z I)\}=\{z \in \mathbb{C}: \mathcal{H} \neq \operatorname{ran}(A-z I) \dot{+} \operatorname{span}\{\kappa\}\} .
$$

The following result is proven in [38, Thm. 2.2]. It was first formulated by Krein without proof in [22, Thm. 8].

Proposition 2.13. For every $A \in \mathcal{S}(\mathcal{H})$, there exists $\kappa$ satisfying (2.7) such that the corresponding set (2.8) does not intersect the real line.

Definition 2.14. We call the vector $\kappa$, whose existence is established in Proposition 2.13, a spectral gauge of $A \in \mathcal{S}(\mathcal{H})$.

Lemma 2.15. A spectral gauge of $A \in \mathcal{S}(\mathcal{H})$ is a generating vector (as defined in [2, Sec. 69]) for any canonical selfadjoint extension of $A$.

Proof. Let $A_{\gamma}$ be any canonical selfadjoint extension of $A$ and $\kappa$ a spectral gauge of $A$. Since $\kappa$ is not in $\operatorname{ran}(A-x I)$ for any $x \in \mathbb{R}, \kappa$ has a nonzero projection onto the one-dimensional space $\operatorname{ker}\left(A^{*}-x I\right)$. Because $\operatorname{ker}\left(A^{*}-x I\right)=\operatorname{ker}\left(A_{\gamma}-x I\right)$ whenever $x \in \operatorname{spec}\left(A_{\gamma}\right)$, it follows that $\kappa$ has a nonzero projection onto every eigenspace of $A_{\gamma}$ since its spectrum is discrete.

The following assertion is stated in [22, Thm. 1] and proven in [15, Thm. 1.2.5].

Proposition 2.16. Let $\kappa$ be a spectral gauge of $A \in \mathcal{S}(\mathcal{H})$ and $a, b \in \mathbb{R}$ such that $a<b$. If $F$ is a generalized spectral family of $A$, then

$$
F([a, b]) \phi=\int_{a}^{b} \frac{\langle\psi(t), \phi\rangle}{\langle\psi(t), \kappa\rangle} d F(t) \kappa
$$

for any $\phi \in \mathcal{H}$ and $\psi$ given in (2.4).

\section{Extremality of measures}

Definition 3.1. Consider $A \in \mathcal{S}(\mathcal{H})$ and let $\kappa$ be a spectral gauge of $A$. Recalling (2.6), define

$$
\mathcal{V}_{\kappa}(A):=\left\{\sigma=\sigma_{A^{+}, \kappa}: A^{+} \text {is selfadjoint extension of } A\right\} .
$$

Inside $\mathcal{V}_{\kappa}(A)$, we single out the set of extremal measures

$$
\mathcal{V}_{\kappa}^{\text {ext }}(A):=\left\{\sigma=\sigma_{A^{+}, \kappa}: A^{+} \text {is canonical selfadjoint extension of } A\right\} .
$$


The reason for using word "extremal" above will be explained in Theorem 3.6 below. In the context of this definition, Proposition 2.7 implies the following.

Corollary 3.2. For any spectral gauge $\kappa$ of $A \in \mathcal{S}(\mathcal{H})$, there is a bijection between $\mathfrak{R}$ and $\mathcal{V}_{\kappa}(A)$. Moreover, this bijection gives a one-to-one mapping between $\mathbb{R} \cup\{\infty\}$ and $\mathcal{V}_{\kappa}^{\text {ext }}(A)$.

The bijection referred above depends on the choice of $A_{\infty}$ (see Section 2.1), but regardless of this choice, all measures arising from spectral families corresponding to canonical selfadjoint extensions of $A$ are obtained when $\tau$ runs through $\mathbb{R} \cup\{\infty\}$. Note that, as a consequence of Proposition 2.12, one has:

Corollary 3.3. Let $\kappa$ be a spectral gauge of $A \in \mathcal{S}(\mathcal{H})$. All measures in $\mathcal{V}_{\kappa}^{\operatorname{ext}}(A)$ are discrete and their supports are pairwise disjoint. For any $x \in \mathbb{R}$, there is a measure $\sigma \in \mathcal{V}_{\kappa}^{\operatorname{ext}}(A)$ such that $\sigma\{x\}>0$.

The next statement can be found in [15, Chap. 2 Sec. 7.1] and the first part of it in [2, Appendix I Sec. 4] (cf. [21, Thm. 6] and [20]).

Theorem 3.4. Consider $A \in \mathcal{S}(\mathcal{H})$. Given $\tau \in \mathfrak{R}$, let $R_{\tau}(z)$ be a generalized resolvent as in (2.5). If $\kappa$ is a spectral gauge of $A$, then

$$
\left\langle\kappa, R_{\tau}(z) \kappa\right\rangle=-\frac{\tau(z) A(z)-C(z)}{\tau(z) B(z)-D(z)},
$$

where $A(z), B(z), C(z), D(z)$ are meromorphic functions on $\mathbb{C}$, analytic on $\mathbb{R}$.

Proof. Reasoning as in [15, Ch. 42, Sec. 7.1], one obtains the stated formula by inserting the right-hand side expression from (2.5) into $\left\langle\kappa, R_{\tau}(z) \kappa\right\rangle$ and considering the functions:

$$
\begin{aligned}
A(z) & :=-\frac{\left\langle\kappa, R_{\infty}(z) \kappa\right\rangle}{\langle\psi(\bar{z}), \kappa\rangle} \\
B(z) & :=\frac{1}{\langle\psi(\bar{z}), \kappa\rangle} \\
C(z) & :=-Q(z) A(z)-\langle\kappa, \psi(z)\rangle \\
D(z) & :=-Q(z) B(z),
\end{aligned}
$$

where $\psi$ is given in (2.4). One verifies from their definition that $A(z), B(z), D(z)$ can be analytically extended to any point $z$ outside the set given in (2.8). The function $C(z)$ has a priori poles in $\operatorname{spec}\left(A_{\infty}\right)$, but by an argument along the lines of $[15$, Ch. 2 Secs. 7.3 and 7.4], one concludes that $C(z)$ can be analytically extended to any point $z$ outside the set given in (2.8). 
Remark 3.5. For Jacobi operators, the functions $A(z), B(z), C(z), D(z)$ coincide with the ones given in [1, Eq. 2.28] and [42, Thm. 4.9]. Note that, for the Herglotz function $-1 / \tau$, [42, Eq. 4.36] is indeed an expression for $\left\langle\kappa, R_{-1 / \tau}(z) \kappa\right\rangle$.

Reasoning along the lines of the proof of [42, Thm. 4.17], one obtains the following assertion from Theorem 3.4.

Theorem 3.6. Let $\kappa$ be a spectral gauge of $A \in \mathcal{S}(\mathcal{H})$ and $\lambda$ a real number. If $\sigma \in \mathcal{V}_{\kappa}(A) \backslash \mathcal{V}_{\kappa}^{\operatorname{ext}}(A)$ is such that $\sigma\{\lambda\}>0$, then there exists $\widetilde{\sigma} \in \mathcal{V}_{\kappa}^{\operatorname{ext}}(A)$ such that $\tilde{\sigma}\{\lambda\}>\sigma\{\lambda\}$.

\section{A generalization of the moment problem}

There are various ways of generalizing the moment problem. We use the generalization proposed by Livsič [28] (see also [44]). According to [28], the generalized moment problem consist in finding a measure $\rho$ such that the inner product in a Hilbert space of functions is expressed through the inner product in $L_{2}(\mathbb{R}, \rho)$. Our choice for the Hilbert space of functions is a de Branges space. In [36, Chap.6], the same choice is done for the generalized moment problem and the treatment is made by means of canonical systems whose theory contains the one of de Branges spaces (see [37]). In contrast to [36, Chap.6], our final goal is the extremal properties of measures.

Definition 4.1. A Hilbert space of entire functions $\mathcal{B}$ is a de Branges space if and only if, for any function $f(z)$ in $\mathcal{B}$, the following conditions holds:

(A1) For all $w \in \mathbb{C}$, the linear functional $f(\cdot) \mapsto f(w)$ is continuous;

(A2) for every non-real zero $w$ of $f(z)$, the function $f(z)(z-\bar{w})(z-w)^{-1}$ belongs to $\mathcal{B}$ and has the same norm as $f(z)$;

(A3) the function $f^{\#}(z):=\overline{f(\bar{z})}$ also belongs to $\mathcal{B}$ and has the same norm as $f(z)$.

Alternatively, a de Branges space can be defined in terms of an HermiteBiehler function, that is, an entire function $e(z)$ such that $|e(z)|>|e(\bar{z})|$ for all $z \in \mathbb{C}_{+}[25$, Ch. 7]. In that case, the de Branges space is sometimes denoted $\mathcal{B}(e)$ and one has

$$
\langle f, g\rangle_{\mathcal{B}}=\int_{\mathbb{R}} \frac{\overline{f(x)} g(x)}{|e(x)|^{2}} d x .
$$

(see [9, Sec. 19], [35, Thm. 2.2], [41]). The r. h.s. of (4.1) is justified by the fact that if $x$ is a real zero of $e(z)$, then $x$ is a zero of greater multiplicity for any function in $\mathcal{B}(e)$. We note that a given de Branges space can be obtained by different Hermite-Biehler functions [8, Thm. 1]. 
Definition 4.2. Given a de Branges space $\mathcal{B}$, the operator $S$ of multiplication by the independent variable is defined by

$$
\operatorname{dom}(S)=\{f(z) \in \mathcal{B}: z f(z) \in \mathcal{B}\} ; \quad(S f)(z)=z f(z), \quad f(z) \in \operatorname{dom}(S) .
$$

It is noteworthy that the operator $S$ is in $\mathcal{S}(\mathcal{B})$ [17, Prop. 4.2, Cor. 4.7]. Moreover, to any operator $A$ in the class $\mathcal{S}(\mathcal{H})$, there corresponds a de Branges space such that the operator of multiplication in it is unitarily equivalent to $A$. To elucidate this, we introduced below the so-called functional model for operators in $\mathcal{S}(\mathcal{H})$.

An involution (conjugation) $J: \mathcal{H} \rightarrow \mathcal{H}$ is an antilinear map satisfying

$$
J^{2}=I \quad \text { and } \quad\langle J \phi, J \psi\rangle=\langle\psi, \phi\rangle
$$

for all $\phi, \psi \in \mathcal{H}[47$, Eq. 8.1]. Fix an operator $A \in \mathcal{S}(\mathcal{H})$ and let $J$ be an involution that commutes with the selfadjoint extensions of $A$ (there is always such involution because of [39, Prop. 2.3]). Consider a function $\xi_{A}: \mathbb{C} \rightarrow \mathcal{H}$ such that

$(\mathrm{P} 1) \xi_{A}(z)$ is zero-free $\left(\xi_{A}(z) \neq 0\right.$ for all $\left.z \in \mathbb{C}\right)$ and entire,

(P2) $\xi_{A}(z) \in \operatorname{ker}\left(A^{*}-z I\right)$ for all $z \in \mathbb{C}$, and

(P3) $J \xi_{A}(z)=\xi_{A}(\bar{z})$ for every $z \in \mathbb{C}$.

Since $A \in \mathcal{S}(\mathcal{H})$, one has $\operatorname{dim} \operatorname{ker}\left(A^{*}-z I\right)=1$ for all $z \in \mathbb{C}$. Using this fact, one can prove the following assertion (see [39, Prop. 2.12 and Remark 2.13]).

Lemma 4.3. If $\xi_{A}^{(1)}: \mathbb{C} \rightarrow \mathcal{H}$ and $\xi_{A}^{(2)}: \mathbb{C} \rightarrow \mathcal{H}$ are two functions satisfying (P1),(P2), and (P3), then there exists a zero-free real entire function $g(z)$ such that $\xi_{A}^{(1)}(z)=g(z) \xi_{A}^{(2)}(z)$.

There is a standard way of constructing a function $\xi_{A}(z)$ with the desired properties: Pick a canonical selfadjoint extension $A_{\gamma}$ of $A \in \mathcal{S}(\mathcal{H})$ and let $h_{\gamma}(z)$ be a real entire function whose zero set (counting multiplicities) equals $\operatorname{spec}\left(A_{\gamma}\right)$; the existence of this function follows from classical theorems on entire functions, see [30, Ch. 7 Sec. 2]. Then, define

$$
\xi_{A}(z):=h_{\gamma}(z) V_{A_{\gamma}}(w, z) \psi_{w},
$$

where $w \in \mathbb{R} \backslash \operatorname{spec}\left(A_{\gamma}\right), \psi_{w} \in \operatorname{ker}\left(A^{*}-w I\right) \backslash\{0\}$ and $V_{A_{\gamma}}(w, z)$ is given by (2.1). It can be shown that (4.2) obeys (P1), (P2) and (P3), the latter relative to a suitable involution [39, Props. 2.3 and 2.11]. Note that Lemma 4.3 implies that (4.2) does not depend on the choice of the selfadjoint extension $A_{\gamma}$ nor on 
$w$ and, furthermore, every function $\xi_{A}: \mathbb{C} \rightarrow \mathcal{H}$ can be written in the form of (4.2).

Fix $A \in \mathcal{S}(\mathcal{H})$ and any function $\xi_{A}: \mathbb{C} \rightarrow \mathcal{H}$ satisfying (P1), (P2), and (P3). Then define the map

$$
\left(\Phi_{A} \varphi\right)(z):=\left\langle\xi_{A}(\bar{z}), \varphi\right\rangle, \quad \varphi \in \mathcal{H}
$$

Due to $(\mathrm{P} 1), \Phi_{A}$ linearly maps $\mathcal{H}$ onto a linear manifold $\Phi_{A} \mathcal{H}$ of entire functions. By [44, Sec. 1], the complete nonselfadjointness condition

$$
\bigcap_{z \in \mathbb{C} \backslash \mathbb{R}} \operatorname{ran}(A-z I)=\{0\}
$$

(see [15, Chap. 1 Thm. 2.1]) implies that $\Phi_{A}$ is injective [41]. Clearly, the linear space $\Phi_{A} \mathcal{H}$ is turned into a Hilbert space by defining

$$
\left\langle\Phi_{A} \eta, \Phi_{A} \varphi\right\rangle:=\langle\eta, \varphi\rangle
$$

The resulting Hilbert space is a de Branges space which is henceforth denoted by $\mathcal{B}_{A}[39$, Prop. 2.14].

Remark 4.4. If $A \in \mathcal{S}(\mathcal{H})$, then $\Phi_{A} A \Phi_{A}^{-1}$ is the multiplication operator $S$ in $\mathcal{B}_{A}$. For any (canonical) selfadjoint extension $A_{\gamma}$ of $A, \Phi_{A} A_{\gamma} \Phi_{A}^{-1}$ is a (canonical) selfadjoint extension of the multiplication operator in $\mathcal{B}_{A}$. Moreover, if $\kappa$ is a spectral gauge of $A$ and $m(z)=\left(\Phi_{A} \kappa\right)(z)$, it can be proven from that

$$
\mathcal{V}_{\kappa}(A)=\mathcal{V}_{m}(S) \quad \text { and } \quad \mathcal{V}_{\kappa}^{\text {ext }}(A)=\mathcal{V}_{m}^{\text {ext }}(S)
$$

Note that, if $F$ is a generalized spectral family of $A$, then $\Phi_{A} F \Phi_{A}^{-1}$ is a generalized spectral family of $S$.

The reproducing kernel in the de Branges space $\mathcal{B}_{A}$ is

$$
k(z, w)=\left\langle\xi_{A}(\bar{z}), \xi_{A}(\bar{w})\right\rangle
$$

Note that $k(z, w)$ is anti-entire with respect to its second argument.

Remark 4.5. It is not difficult to verify that $k(\cdot, w) \in \operatorname{ker}\left(S^{*}-\bar{w} I\right)$ for all $w \in \mathbb{C}$ and moreover

$$
k(z, w)=h(\bar{w}) V_{S_{\gamma}}(\bar{v}, \bar{w}) k(z, v)
$$

where $S_{\gamma}$ is a canonical selfadjoint extension of $S$ given in Definition 4.2 and $h(w)$ is a real entire function having zeros at $\operatorname{spec}\left(S_{\gamma}\right)(c f .(2.4)$ and (4.2)). 
Lemma 4.6. If $\kappa$ is a spectral gauge of $A \in \mathcal{S}(\mathcal{H})$, then the corresponding element $m$ in $\mathcal{B}_{A}$ (given by Remark 4.4) has no zeros in $\mathbb{R}$. Moreover, if the function $m$ in the de Branges space $\mathcal{B}$ is such that $m(x) \neq 0$ for all $x \in \mathbb{R}$, then $m$ is a spectral gauge of the operator of multiplication $S$ in $\mathcal{B}$.

Proof. By our definition of spectral gauge,

$$
\langle\xi(\bar{z}), \kappa\rangle \neq 0
$$

for any $z \in \mathbb{R}$. The second part of the statement follows from noticing that $k(\cdot, w) \in \operatorname{ker}\left(S^{*}-\bar{w} I\right)$ for all $w \in \mathbb{C}$.

The following definition coincides with [36, Def.6.1]. We came to it motivated by a generalization of the moment problem discussed in [28] (see also [44]). Several results in [9] pertain to this definition.

Definition 4.7 (Generalized moment problem). Given a de Branges space $\mathcal{B}$, find a Borel measure $\rho$ such that

$$
\langle g, f\rangle_{\mathcal{B}}=\int_{\mathbb{R}} f(x) \overline{g(x)} d \rho(x)
$$

for every $f, g \in \mathcal{B}$.

As (4.1) shows, the Lebesgue measure multiplied by $1 /|e(\cdot)|^{2}$ is always a solution to the generalized moment problem for $\mathcal{B}(e)$. Below, it will be established that there are other solutions to this generalized moment problem.

Theorem 4.8. Let $S$ be the operator of multiplication by the independent variable in a de Branges space $\mathcal{B}$ and $m$ be a function in $\mathcal{B}$ not vanishing in $\mathbb{R}$. If $\rho$ is a solution to the generalized moment problem for $\mathcal{B}$, then

$$
\sigma(\partial):=\int_{\partial}|m(x)|^{2} d \rho(x), \quad \partial \in \mathfrak{X}
$$

belongs to $\mathcal{V}_{m}(S)$.

Proof. Fix $t \in \mathbb{R}$ and define

$$
\Upsilon_{t}(f, g):=\int_{-\infty}^{t} f(x) \overline{g(x)} d \rho(x)
$$

which is a sesquilinear form in $\mathcal{B} \times \mathcal{B}$. This form is bounded due to the inequality

$$
\Upsilon_{t}(f, f) \leq \int_{\mathbb{R}}|f(x)|^{2} d \rho(x)=\|f\|^{2}
$$


along with the polarization identity. By [6, Ch. 2 Sec. 4 Thm. 6] and well-known results on sesquilinear forms [34, Sec. II.2], there is a bounded operator $F(t)$ defined on the whole space $\mathcal{B}$ such that

$$
\langle g, F(t) f\rangle=\Upsilon_{t}(f, g) .
$$

It turns out that $F(t)$ corresponds to a generalized spectral family in the sense of Proposition 2.6. Indeed, (i)-(iii) are verified directly from the definition. As regards (iv), one verifies

$$
\int_{\mathbb{R}} t d\langle g, F(t) f\rangle=\int_{\mathbb{R}} t f(t) \overline{g(t)} d \rho(t)=\langle g, S f\rangle_{\mathcal{B}}
$$

and

$$
\int_{\mathbb{R}} t^{2} d\langle f, F(t) f\rangle=\int_{\mathbb{R}} t^{2} f(t) \overline{f(t)} d \rho(t)=\langle S f, S f\rangle_{\mathcal{B}} .
$$

Thus, for any real Borel set $\partial$,

$$
\mathcal{V}_{m}(S) \ni\langle m, F(\partial) m\rangle=\int_{\partial}|m(x)|^{2} d \rho(x)
$$

since $m$ is a spectral gauge of $S$ by Lemma 4.6 .

Lemma 4.9. Let $m \in \mathcal{B}$ be such that $m(x) \neq 0$ when $x \in \mathbb{R}$ and $a, b \in \mathbb{R}$ such that $a<b$. If $F$ is a generalized spectral family of $S$, then

$$
(F([a, b]) f)(z)=\int_{a}^{b} \frac{f(t)}{m(t)} d F(t) m(z)
$$

for any $f \in \mathcal{B}$.

Proof. According to Remark 4.5, since $m(z)$ is a spectral gauge of $S$, the righthand side of (2.9) in this case is

$$
\int_{a}^{b} \frac{\langle k(\cdot, t), f(\cdot)\rangle_{\mathcal{B}}}{\langle k(\cdot, t), m(\cdot)\rangle_{\mathcal{B}}} d F(t) m(z) .
$$

The assertion then follows from Proposition 2.16.

Theorem 4.10. Let $S$ be the operator of multiplication by the independent variable in a de Branges space $\mathcal{B}$ and $m$ be a function in $\mathcal{B}$ not vanishing in $\mathbb{R}$. If $\sigma \in \mathcal{V}_{m}(S)$, then

$$
\rho(\partial):=\int_{\partial} \frac{d \sigma(x)}{|m(x)|^{2}}, \quad \partial \in \mathfrak{X}
$$

is a solution to the generalized moment problem for $\mathcal{B}$. 
Proof. By the hypothesis and Lemma 4.9,

$$
\sigma(\partial)=\langle m, F(\partial) m\rangle_{\mathcal{B}}
$$

for any real Borel set $\partial$. It follows from Lemma 4.9 that

$$
\langle g, F[a, b] f\rangle_{\mathcal{B}}=\int_{a}^{b} \frac{f(t)}{m(t)} d\langle g, F(t) m\rangle_{\mathcal{B}}
$$

Also

$$
\langle F(t) g, m\rangle_{\mathcal{B}}=\langle F(a) g, m\rangle_{\mathcal{B}}+\int_{a}^{t} \frac{\overline{g(x)}}{\overline{m(x)}} d\langle F(x) m, m\rangle_{\mathcal{B}}
$$

Putting the last two equalities together, one obtains

$$
\langle g, F[a, b] f\rangle_{\mathcal{B}}=\int_{a}^{b} \frac{f(t) \overline{g(t)}}{|m(t)|^{2}} d \sigma(t)=\int_{a}^{b} f(t) \overline{g(t)} d \rho(t)
$$

For finishing the proof take the limit when $a \rightarrow-\infty$ and $b \rightarrow+\infty$.

Lemma 4.11. Let $S$ be the operator of multiplication by the independent variable in a de Branges space $\mathcal{B}$ and $m$ be a function in $\mathcal{B}$ not vanishing in $\mathbb{R}$. If $S_{\gamma}$ is a canonical selfadjoint extension of $S$ and

$$
\sigma(\partial):=\left\langle m, E_{\gamma}(\partial) m\right\rangle, \quad \partial \in \mathfrak{X}
$$

where $E_{\gamma}$ is the spectral family of $S_{\gamma}$, then

$$
\left(\mathfrak{f}\left(S_{\gamma}\right) m\right)(\lambda)=\mathfrak{f}(\lambda) m(\lambda)
$$

for any $\mathfrak{f}$ in $L_{2}(\mathbb{R}, \sigma)$ and $\lambda \in \operatorname{spec}\left(S_{\gamma}\right)$.

Proof. Using the spectral theorem,

$$
\left(\mathfrak{f}\left(S_{\gamma}\right) m\right)(z)=\left(\int_{\mathbb{R}} \mathfrak{f}(t) d E_{\gamma}(t) m\right)(z)=\sum_{\lambda \in \operatorname{spec}\left(S_{\gamma}\right)} \mathfrak{f}(\lambda) k(z, \lambda) \frac{\langle k(\cdot, \lambda), m\rangle_{\mathcal{B}}}{k(\lambda, \lambda)} .
$$

From the last expression the result follows due to the properties of the reproducing kernel.

Theorem 4.12. Let $\mathcal{B}, S$ and $m$ be as in the previous theorem. Assume that $\rho$ is a solution to the generalized moment problem and $\sigma$ is related to it as in Theorem 4.10. A necessary and sufficient condition for the measure $\sigma$ to be in $\mathcal{V}_{m}^{\text {ext }}(S)$ is that the map $\Psi$ between the spaces $\mathcal{B}$ and $L_{2}(\mathbb{R}, \rho)$ given by

$$
\Psi f=f \uparrow_{\mathbb{R}}
$$


is unitary (that is, linear, surjective and norm-preserving).

Proof. Since $\rho$ is a solution to the generalized moment problem, $\Psi \mathcal{B}$ is contained in $L_{2}(\mathbb{R}, \rho)$, where $\Psi f=f \uparrow_{\mathbb{R}}$ and $\Psi$ is isometric. The hypothesis $\left\{f \uparrow_{\mathbb{R}}: f \in\right.$ $\mathcal{B}\}=L_{2}(\mathbb{R}, \rho)$ therefore means that $\Psi$ is unitary. Let $A^{\rho}$ be the operator of multiplication by the independent variable in $L_{2}(\mathbb{R}, \rho)$ defined in the maximal domain. Since $A^{\rho}$ is selfadjoint, the same holds for $\Psi^{-1} A^{\rho} \Psi$. If $f \in \operatorname{dom}(S)$, then $\Psi f \in \operatorname{dom}\left(A^{\rho}\right)$. Thus, $\Psi^{-1} A^{\rho} \Psi$ is a canonical selfadjoint extension of $S$. Denote $S_{\gamma}:=\Psi^{-1} A^{\rho} \Psi$ and let $E_{\gamma}$ be the spectral family of $S_{\gamma}$. For any Borel set $\partial \subset \mathbb{R}$, define $\eta(\partial):=\left\langle m, E_{\gamma}(\partial) m\right\rangle$. By the canonical map (see [2, Sec. 69]),

$$
L_{2}(\mathbb{R}, \eta) \ni \mathfrak{f} \mapsto \mathfrak{f}\left(S_{\gamma}\right) m \in \mathcal{B}
$$

and for any function $f$ in $\mathcal{B}$ there is a function $\mathfrak{f}$ in $L_{2}(\mathbb{R}, \eta)$ such that $f=\mathfrak{f}\left(S_{\gamma}\right) m$. Thus

$$
\int_{\mathbb{R}} f(t) \overline{g(t)} d \rho(t)=\int_{\mathbb{R}} \mathfrak{f}(t) \overline{\mathfrak{g}(t)} d \eta(t),
$$

where $f$ and $g$ are the images of $\mathfrak{f}$ and $\mathfrak{g}$ under the canonical map. On the basis of Lemma 4.11, $f(t)=\mathfrak{f}(t) m(t)$ and $g(t)=\mathfrak{g}(t) m(t)$ with the equalities in the $L_{2}(\mathbb{R}, \eta)$ sense. Therefore

$$
\int_{\mathbb{R}} f(t) \overline{g(t)} d \rho(t)=\int_{\mathbb{R}} f(t) \overline{g(t)} \frac{d \eta(t)}{|m(t)|^{2}}
$$

Since this equality holds for any element in $L_{2}(\mathbb{R}, \rho)$, one concludes that $\eta(\partial)=$ $\sigma(\partial)$ for any real Borel set $\partial$ and hence $\sigma$ is extremal, that is, $\sigma \in \mathcal{V}_{m}^{\text {ext }}(S)$.

Let us prove the other direction. If $\sigma \in \mathcal{V}_{m}^{\text {ext }}(S)$, then $\sigma$ is the spectral measure of a canonical selfadjoint extension of $S$ which is known to be simple. By the canonical map (see [2, Sec. 69]), $\mathcal{B}$ is unitarily equivalent to $L_{2}(\mathbb{R}, \sigma)$. In turn, since $m$ is zero-free on the real axis, $L_{2}(\mathbb{R}, \sigma)$ is unitarily equivalent to $L_{2}(\mathbb{R}, \rho)$. On the other hand, the Hilbert spaces $\mathcal{B}$ and $\Psi \mathcal{B} \subset L_{2}(\mathbb{R}, \rho)$ are unitarily equivalent due to the fact that $\rho$ is a solution to the moment problem. Therefore, $\Psi \mathcal{B}$ cannot be properly contained in $L_{2}(\mathbb{R}, \rho)$.

Definition 4.13. A solution $\rho$ to the generalized moment problem for $\mathcal{B}$ is said to be extremal if the map $\Psi: \mathcal{B} \rightarrow L_{2}(\mathbb{R}, \rho)$ given in Theorem 4.12 is unitary.

Unlike the extremal measures (see Definition 3.1), the extremal solutions might not be finite measures. However, the extremal solutions of the generalized moment problem for $\mathcal{B}$ can be finite measures if, for instance, $1 \in \mathcal{B}$ (see Example 6.1).

Let $\delta_{x}: \mathfrak{X} \rightarrow[0,1]$ be the measure defined by the rule $\delta_{x}(\partial):= \begin{cases}1 & x \in \partial \\ 0 & x \notin \partial .\end{cases}$ 
Theorem 4.14. Let $\mathcal{B}$ be a de Branges space, $k(\cdot, w)$ its reproducing kernel, and $S_{\gamma}$ a canonical selfadjoint extension of the operator of multiplication $S$. The measure

$$
\rho_{\gamma}:=\sum_{\lambda \in \operatorname{spec}\left(S_{\gamma}\right)} \frac{\delta_{\lambda}}{k(\lambda, \lambda)}
$$

is an extremal solution to the generalized moment problem for $\mathcal{B}$. Reciprocally, every extremal solution is of the form (4.3).

Proof. It follows from Remark 4.5 and the equality $\operatorname{ker}\left(S_{\gamma}-\lambda I\right)=\operatorname{ker}\left(S^{*}-\lambda I\right)$ that $\{k(\cdot, \lambda) /\|k(\cdot, \lambda)\|\}_{\lambda \in \operatorname{spec}\left(S_{\gamma}\right)}$ is an orthonormal basis in $\mathcal{B}$. Therefore, on the one hand, a function $f$ is in $\mathcal{B}$ if and only if

$$
\sum_{\lambda \in \operatorname{spec}\left(S_{\gamma}\right)} \frac{|f(\lambda)|^{2}}{k(\lambda, \lambda)}<+\infty
$$

and, on the other hand, one has the following interpolation formula

$$
f(z)=\sum_{\lambda \in \operatorname{spec}\left(S_{\gamma}\right)} \frac{k(z, \lambda)}{k(\lambda, \lambda)} f(\lambda)
$$

for every $f \in \mathcal{B}$, where the convergence is in the sense of Hilbert space, hence uniform in compact subsets of $\mathbb{C}$. Thus, if $\rho_{\gamma}$ is given by (4.3), then every $f$ in $L_{2}\left(\mathbb{R}, \rho_{\gamma}\right)$ satisfies (4.4) and, through the interpolation formula (4.5), it is mapped into a function in $\mathcal{B}$ whose restriction to the real axis is the function $f$. The identity

$$
\|f\|_{\mathcal{B}}^{2}=\sum_{\lambda \in \operatorname{spec}\left(S_{\gamma}\right)} \frac{|f(\lambda)|^{2}}{k(\lambda, \lambda)}=\|f\|_{L_{2}\left(\mathbb{R}, \rho_{\gamma}\right)}^{2} .
$$

implies that $\Psi$ is norm-preserving.

Conversely, if $\rho$ is an extremal solution, then $\operatorname{supp} \rho$ coincides with $\operatorname{spec}\left(S_{\gamma}\right)$ for some $\gamma$ as a consequence of Theorem 4.12. Thus, for any $f$ in $L_{2}(\mathbb{R}, \rho)$ whose zero set is $\operatorname{spec}\left(S_{\gamma}\right) \backslash\{\lambda\}$, one has

$$
\frac{|f(\lambda)|^{2}}{k(\lambda, \lambda)}=\|f\|_{\mathcal{B}}^{2}=\|f\|_{L_{2}(\mathbb{R}, \rho)}^{2}=c|f(\lambda)|^{2}
$$

which shows that the weight $c$ of the measure $\rho$ at $\lambda$ should be equal to $1 / k(\lambda, \lambda)$.

Remark 4.15. Assume that $A \in \mathcal{S}(\mathcal{H})$ and take the measure $\rho_{\gamma}$ as defined in Theorem 4.14 for the corresponding de Branges space $\mathcal{B}_{A}$. The multiplication by the independent variable in $L_{2}\left(\mathbb{R}, \rho_{\gamma}\right)$ is unitarily equivalent to a canonical 
selfadjoint extension $A_{\gamma}$ of $A$. Note that one can write

$$
\rho_{\gamma}=\sum_{\lambda \in \operatorname{spec}\left(S_{\gamma}\right)} \frac{\delta_{\lambda}}{\left\|\xi_{A}(\lambda)\right\|^{2}} .
$$

It is worth remarking that in the Sturm-Liouville theory (when $A$ is associated with a regular Sturm-Liouville difference expression) the measure $\rho_{\gamma}$ given above is usually defined via the Weyl function and is called the spectral measure of the selfadjoint operator $A_{\gamma}$ (see details in Example 6.2).

\section{Point mass perturbations of measures}

Lemma 5.1. Let $\mathcal{B}$ be a de Branges space with inner product $\langle\cdot, \cdot\rangle_{\mathcal{B}}$. Given $a>0$ and $\lambda \in \mathbb{R}$, define

$$
\langle g, f\rangle_{\sim}:=\langle g, f\rangle_{\mathcal{B}}+a \overline{g(\lambda)} f(\lambda), \quad f, g \in \mathcal{B} .
$$

Then the linear manifold $\mathcal{B}$ equipped with the inner product $\langle\cdot, \cdot\rangle_{\sim}$ is a de Branges space.

Proof. $\mathcal{B}$ is closed with respect to $\langle\cdot, \cdot\rangle_{\sim}$. Indeed, let $\left\{f_{n}\right\}_{0}^{\infty} \subset \mathcal{B}$ be a $\sim$-Cauchy sequence. Since $\|\cdot\|_{\sim} \geq\|\cdot\|_{\mathcal{B}},\left\{f_{n}\right\}_{0}^{\infty}$ is also $\mathcal{B}$-Cauchy. Then, there exists $g \in \mathcal{B}$ such that $\left\|f_{n}-g\right\|_{\mathcal{B}} \rightarrow 0$. Since $\left|f_{n}(\lambda)-g(\lambda)\right|^{2} \leq k(\lambda, \lambda)\left\|f_{n}-g\right\|_{\mathcal{B}}^{2}$, one obtains

$$
\left\|f_{n}-g\right\|_{\sim}^{2} \leq\left\|f_{n}-g\right\|_{\mathcal{B}}^{2}+a k(\lambda, \lambda)\left\|f_{n}-g\right\|_{\mathcal{B}}^{2},
$$

implying the assertion.

Now we prove (A1)-(A3) of Definition 4.1. Since

$$
|f(w)-g(w)|^{2}=|\langle k(\cdot, w), f-g\rangle|^{2} \leq k(w, w)\|f-g\|_{\mathcal{B}}^{2} \leq k(w, w)\|f-g\|_{\sim}^{2},
$$

it follows that point evaluation is continuous with respect to the $\sim$-norm.

From the equality

$$
\left\|f^{\#}\right\|_{\sim}^{2}=\left\|f^{\#}\right\|_{\mathcal{B}}^{2}+a\left|f^{\#}(\lambda)\right|^{2}=\|f\|_{\mathcal{B}}^{2}+a|f(\lambda)|^{2}=\|f\|_{\sim}^{2},
$$

it follows that the mapping $f \mapsto f^{\#}$ (see (A3) in Definition 4.1) is a $\sim$-isometry in $\mathcal{B}$.

Finally, suppose $w \in \mathbb{C} \backslash \mathbb{R}$ is a zero of $f \in \mathcal{B}$ and define $g(z):=(z-\bar{w})(z-$ $w)^{-1} f(z)$. Then, $g \in \mathcal{B}$ and

$$
\|g\|_{\sim}^{2}=\|g\|_{\mathcal{B}}^{2}+a\left|\frac{\lambda-\bar{w}}{\lambda-w}\right|^{2}|f(x)|^{2}=\|f\|_{\mathcal{B}}^{2}+a|f(x)|^{2}=\|f\|_{\sim}^{2} .
$$


Lemma 5.1 has the following corollary.

Corollary 5.2. Let $\mathcal{B}$ be a de Branges space. If $\rho$ is a solution to the generalized moment problem for $\mathcal{B}$, then $\rho+a \delta_{\lambda}(a>0, \lambda \notin \operatorname{supp} \rho)$ is a solution to the generalized moment problem for a de Branges space $\mathcal{B}_{\sim}$ having the same elements as $\mathcal{B}$ but with inner product given by (5.1).

Proof. The statement follows from the equality

$$
\int_{\mathbb{R}} \overline{g(x)} f(x) d\left(\rho+a \delta_{\lambda}\right)=\langle g, f\rangle_{\mathcal{B}}+a \overline{g(\lambda)} f(\lambda) .
$$

Theorem 5.3. Let $S$ be the operator of multiplication by the independent variable in a de Branges space $\mathcal{B}$ and $m$ be a function in $\mathcal{B}$ not vanishing in $\mathbb{R}$. If $\sigma \in \mathcal{V}_{m}(S)$, then

$$
\sigma+a|m(\lambda)|^{2} \delta_{\lambda}, \quad a>0, \quad \lambda \notin \operatorname{supp}(\sigma),
$$

is in $\mathcal{V}_{m}(\widetilde{S}) \backslash \mathcal{V}_{m}^{\text {ext }}(\widetilde{S})$, where $\widetilde{S}$ is the multiplication operator in the de Branges space $\mathcal{B}_{\sim}$ given in Corollary 5.2.

Proof. By hypothesis, $\rho$ given in Theorem 4.10 is a solution to the generalized moment problem for $\mathcal{B}$. By Corollary 5.2, $\rho+a \delta_{\lambda}$ is a solution to the generalized moment problem for $\mathcal{B}_{\sim}$ and Theorem 4.8 implies that

$$
\sigma+a|m(\lambda)|^{2} \delta_{\lambda} \in \mathcal{V}_{m}(\widetilde{S})
$$

On the other hand, Corollary 3.3 yields a measure $\sigma_{\lambda} \in \mathcal{V}_{m}^{\text {ext }}(S)$ such that $\sigma_{\lambda}\{\lambda\}>0$. The function $\rho_{\lambda}$ related to $\sigma_{\lambda}$ as in Theorem 4.10 is a solution to the generalized moment problem for $\mathcal{B}$ and recurring again to Corollary 5.2, one concludes that $\rho_{\lambda}+a \delta_{\lambda}$ is a solution to the generalized moment problem for $\mathcal{B}_{\sim}$. Thus, in view of Theorem 4.8,

$$
\sigma_{\lambda}+a|m(\lambda)|^{2} \delta_{\lambda} \in \mathcal{V}_{m}(\widetilde{S})
$$

Since $\lambda \in \operatorname{supp}\left(\sigma_{\lambda}\right) \backslash \operatorname{supp}(\sigma)$, one has

$$
\left(\sigma_{\lambda}+a|m(\lambda)|^{2} \delta_{\lambda}\right)\{\lambda\}>\left(\sigma+a|m(\lambda)|^{2} \delta_{\lambda}\right)\{\lambda\}>0 .
$$

Assume that $\sigma+a|m(\lambda)|^{2} \delta_{\lambda}$ is extremal. If $\sigma_{\lambda}+a|m(\lambda)|^{2} \delta_{\lambda}$ is extremal, then a contradiction arises from Corollary 3.3. If $\sigma_{\lambda}+a|m(\lambda)|^{2} \delta_{\lambda}$ is not extremal, then, using Theorem 3.6, one obtains an extremal measure giving weight to $\lambda$ so again we have a contradiction by Corollary 3.3. Therefore $\sigma+a|m(\lambda)|^{2} \delta_{\lambda}$ is not in $\mathcal{V}_{m}^{\text {ext }}(\widetilde{S})$. 
Theorem 5.4. Let $\mathcal{B}$ and $\mathcal{B}^{\prime}$ be de Branges spaces such that they are set-wise equal. If $\rho$ is a solution to the generalized moment problem for $\mathcal{B}$, then $\rho+a \delta_{\lambda}$ $(a>0, \lambda \notin \operatorname{supp} \rho)$ is not an extremal solution to the generalized moment problem for $\mathcal{B}^{\prime}$.

Remark 5.5. Before proving the assertion, there are two points to comment. First, the measure $\rho+a \delta_{\lambda}$ is not necessarily a solution of the generalized moment problem for $\mathcal{B}^{\prime}$. Second, the particular case when in the hypothesis $\rho$ is an extremal solution has interesting applications (see Corollary 5.7 and Section 6).

Proof. Assume that $\rho+a \delta_{\lambda}$ is an extremal solution to the generalized moment problem for $\mathcal{B}^{\prime}$. Thus,

$$
\|f\|_{\mathcal{B}^{\prime}}^{2}=\int_{\mathbb{R}}|f(x)|^{2} d \rho+a|f(\lambda)|^{2}
$$

By hypothesis,

$$
\|f\|_{\mathcal{B}}^{2}=\int_{\mathbb{R}}|f(x)|^{2} d \rho .
$$

Therefore the inner products of $\mathcal{B}$ and $\mathcal{B}^{\prime}$ are related as in (5.1). Now, since $\rho$ and $\rho+a \delta_{\lambda}$ generate via Theorem 4.8 spectral measures of the operators of multiplication in $\mathcal{B}$ and $\mathcal{B}^{\prime}$, one obtains a contradiction from Theorem 5.3.

Remark 5.6. There is an alternative proof of Theorem 5.4. Indeed, assume that $\rho+a \delta_{\lambda}$ is an extremal solution of the moment problem for $\mathcal{B}^{\prime}$ and consider a function $g \in L_{2}\left(\mathbb{R}, \rho+a \delta_{\lambda}\right)$ whose zero set is $\operatorname{supp} \rho$. Thus, $\|g\|_{L_{2}\left(\mathbb{R}, \rho+a \delta_{\lambda}\right)} \neq 0$ and, since $\Psi$ is surjective and norm preserving, the function $\Psi^{-1} g$ is a nonzero element of $\mathcal{B}^{\prime}$ and therefore a nonzero element of $\mathcal{B}$. But we get a contradiction since the norm of the restriction to the real line of $\Psi^{-1} g$ has zero norm in $L_{2}(\mathbb{R}, \rho)$.

Corollary 5.7. Let $\rho$ be a solution to the generalized moment problem for a de Branges space $\mathcal{B}$. If $\mathcal{A} \subset \Psi \mathcal{B}$ is dense in $L_{2}(\mathbb{R}, \rho)$, then $\mathcal{A}$ is not dense in $L_{2}\left(\mathbb{R}, \rho+a \delta_{\lambda}\right)$, where $a>0$ and $x \notin \operatorname{supp}(\rho)$.

Proof. The hypothesis implies that $\rho$ is an extremal solution, i. e. $\Psi \mathcal{B}=L_{2}(\mathbb{R}, \rho)$. As in Corollary 5.2, let $\mathcal{B}_{\sim}$ be the de Branges space having the same elements of $\mathcal{B}$ with the inner product given by (5.1). Then $\Psi \mathcal{B}_{\sim}$, which contains $\mathcal{A}$, is not dense in $L_{2}\left(\mathbb{R}, \rho+a \delta_{\lambda}\right)$ as a consequence of Theorem 5.4.

Remark 5.8. Note that a function is square-integrable with respect to $\rho$ if and only if it is square-integrable with respect to $\rho+a \delta_{\lambda}$. Nevertheless, there functions in the equivalence class of zero in $L_{2}(\mathbb{R}, \rho)$ which have nonzero norm in $L_{2}(\mathbb{R}, \rho)$. 


\section{Examples}

\subsection{The classical moment problem}

According to [1, Thm. 2.1.1], for a given real sequence $\left\{s_{k}\right\}_{k=0}^{\infty}$, there exists a Borel measure $\rho$ such that

$$
s_{k}=\int_{\mathbb{R}} t^{k} d \rho(t) \quad \text { for } k=0,1, \ldots
$$

if and only if, for all $k \in \mathbb{N}$,

$$
\operatorname{det}\left(\begin{array}{ccc}
s_{0} & \ldots & s_{k} \\
\vdots & \vdots & \vdots \\
s_{k} & \ldots & s_{2 k}
\end{array}\right)>0 .
$$

The measure is said to be a solution to the moment problem given by the sequence $\left\{s_{k}\right\}_{k=0}^{\infty}$. We refer to all sequences $\left\{s_{k}\right\}_{k=0}^{\infty}$ satisfying (6.1) and normalized so that $s_{0}=1$ as sequences of moments. To any sequence of moments there corresponds one and only one Jacobi matrix

$$
\left(\begin{array}{ccccc}
q_{1} & b_{1} & 0 & 0 & \cdots \\
b_{1} & q_{2} & b_{2} & 0 & \\
0 & b_{2} & q_{3} & b_{3} & \ddots \\
0 & 0 & b_{3} & q_{4} & \ddots \\
\vdots & & \ddots & \ddots & \ddots
\end{array}\right),
$$

where $\left\{q_{k}\right\}_{k=1}^{\infty}$ is a sequence of real numbers and $\left\{b_{k}\right\}_{k=1}^{\infty}$ is a sequence of positive numbers (see [1, paragraph after Eq. 1.8] and [42, p. 93]). Given a separable Hilbert space $\mathcal{H}$ and an orthonormal basis $\left\{\delta_{k}\right\}_{k=1}^{\infty}$, with any matrix (6.2), one can uniquely associate a closed symmetric operator [2, Sec. 47]. This operator, denoted by $A$ and called minimal Jacobi operator, has either deficiency indices $n_{+}(A)=n_{-}(A)=0$ or $n_{+}(A)=n_{-}(A)=1[5$, Ch. 7, Thm. 1.1]. When the Jacobi operator has deficiency indices $(0,0)$ the matrix $(6.2)$ is said to be in the limit point case, otherwise the matrix (6.2) is said to be in the limit circle case. Due to the bijection between Jacobi matrices and sequences of moments, the limit point and limit circle dichotomy corresponds to the determinate and indeterminate dichotomy for sequences of moments.

It is established in [1, Ch. 4] that

$$
s_{k}=\left\langle\delta_{1}, A^{k} \delta_{1}\right\rangle \quad \text { for } k=0,1, \ldots
$$


This shows that if $A$ has deficiency indices $n_{+}(A)=n_{-}(A)=1$, then the spectral measures of canonical selfadjoint extensions are solutions of the moment problem, $i$. e. an indeterminate sequence of moments admits various solutions. According to $\left[1\right.$, Cor. 2.2.4], when the deficiency indices $n_{+}(A)$ and $n_{-}(A)$ vanish, there are only one solution to the moment problem. This justifies the terminology.

A Jacobi operator $A$ with deficiency indices $n_{+}(A)=n_{-}(A)=1$ is in $\mathcal{S}(\mathcal{H})$. Indeed, if one assume that $\lambda \in \widehat{\operatorname{spec}}(A)$, then, since the spectral kernel does not decrease under extensions, $\lambda \in \operatorname{spec}\left(A_{\gamma}\right)$ for any selfadjoint extension $A_{\gamma}$ of $A$. But the spectra of canonical selfadjoint extensions of $A$ are disjoint (see the proof of [1, Thm. 4.2.4].

For a Jacobi operator $A \in \mathcal{S}(\mathcal{H})$, the function $\xi_{A}$ defined in (4.2) is given by $\xi_{A}(z)=\sum_{k=1}^{\infty} P_{k-1}(z) \delta_{k}$, where $P_{k}$ is the $\mathrm{k}$-th orthogonal polynomial of the first kind associated with (6.2) (see [1, Ch. 1 Sec. 2, Ch. 4 Sec. 1] and [5, Ch. 7 Sec. 2 ]). Thus we have the unitary map

$$
\Phi_{A}: \mathcal{H} \rightarrow \mathcal{B}_{A}
$$

Note first that, since the finite linear combinations of the basis $\left\{\delta_{k}\right\}_{k=1}^{\infty}$ are dense in $\mathcal{H}$, the polynomials are dense in $\mathcal{B}_{A}$. This is a peculiarity of de Branges spaces generated by Jacobi operators. Also,

$$
\left(\Phi_{A} A \delta_{1}\right)(z)=\left\langle\xi_{A}(\bar{z}), A \delta_{1}\right\rangle=\left\langle A^{*} \xi_{A}(\bar{z}), \delta_{1}\right\rangle=\left\langle\bar{z} \xi_{A}(\bar{z}), \delta_{1}\right\rangle=z
$$

since $\left\langle\xi_{A}(\bar{z}), \delta_{1}\right\rangle=1$. Thus

$$
\left(\Phi_{A} A^{k} \delta_{1}\right)(z)=z^{k} \quad \text { for all } k \in \mathbb{N} \cup\{0\} .
$$

Theorem 6.1. The measure $\rho$ is a solution to an indeterminate moment problem if and only if $\rho$ is a solution to the generalized moment problem for $\mathcal{B}_{A}$.

Proof. Assume that $\rho$ is a solution to the generalized moment problem for $\mathcal{B}_{A}$. Then, in view of (6.3) and (6.4), one has

$$
s_{k}=\left\langle 1, z^{k}\right\rangle_{\mathcal{B}_{A}}=\int_{\mathbb{R}} t^{k} d \rho(t) \quad \text { for } k=0,1, \ldots
$$

which means that $\rho$ is a solution to the moment problem given by $\left\{s_{k}\right\}_{k=0}^{\infty}$.

Now, suppose that $\rho$ is a solution to the moment problem given by $\left\{s_{k}\right\}_{k=0}^{\infty}$. Hence, by (6.3), one has

$$
\int_{\mathbb{R}} t^{k} d \rho(t)=\left\langle\delta_{1}, A^{k} \delta_{1}\right\rangle \quad \text { for } k=0,1, \ldots
$$


One then verifies that if $R(t)$ is a polynomial, then

$$
\int_{\mathbb{R}} R(t) d \rho(t)=\left\langle\delta_{1}, R(A) \delta_{1}\right\rangle
$$

Thus

$$
\int_{\mathbb{R}}|R(t)|^{2} d \rho(t)=\left\langle R(A) \delta_{1}, R(A) \delta_{1}\right\rangle
$$

But, due to (6.4),

$$
\left\langle R(A) \delta_{1}, R(A) \delta_{1}\right\rangle=\langle R, R\rangle_{\mathcal{B}_{A}}
$$

For completing the proof one uses the fact that the polynomials are dense in $\mathcal{B}_{A}$ and the polarization identity.

In the context of the classical moment problem, Theorem 5.3 corresponds to [10, Prop. 4.1(a)], which says that if $\rho$ is an extremal solution to an indeterminate moment problem, then $\rho+a \delta_{\lambda}(a>0, \lambda \notin \operatorname{supp} \rho)$ is not extremal although is a solutions of a moment problem. Thus, according to Corollary 5.7, the density of the polynomials in $L_{2}(\mathbb{R}, \rho)$ no longer holds in $L_{2}\left(\mathbb{R}, \rho+a \delta_{\lambda}\right)$. Note that the sequences of moments for which $\rho$ and $\rho+a \delta_{\lambda}$ are different, therefore the corresponding Jacobi operators are different and the corresponding de Branges spaces are different. However, the associated de Branges spaces are set-wise equal, which is actually a consequence of the fact that the set of polynomials are dense in these two spaces, as well as the argument in the proof of Lemma 5.1.

We conclude this example by pointing out that it was probably first mentioned in [14, Remark 4.5 (iii)] that the set of points obtained by adding a point to the spectrum of a selfadjoint extension of a discrete Schrödinger operator in the limit circle case is no longer the spectrum of a selfadjoint extension of a discrete Schrödinger operator in the limit circle case (cf. [13, Sec. 7 Example 2] and [26, Chap. 2 Sec. 7]).

\subsection{Schrödinger operators with measures}

The following example is based on [4] (see also [29]). Let us consider the differential expression given informally by

$$
\tau_{\mu}:=-\frac{d^{2}}{d x^{2}}+\mu, \quad x \in[0, b]
$$

where $\mu$ is a signed Borel measure on $[0, b]$. Properly, for a function $\varphi$ in $A C[0, b]$ we define

$$
\varphi^{[1]}(x):=\varphi^{\prime}(x)-\int_{[0, x]} \varphi(t) d \mu(t)
$$


then we define

$$
\begin{gathered}
\operatorname{dom}\left(\tau_{\mu}\right):=\left\{\varphi \in A C[0, b]: \varphi^{[1]} \in A C[0, b]\right\} \\
\tau_{\mu} \varphi:=-\left(\varphi^{[1]}\right)^{\prime} .
\end{gathered}
$$

The derivative of an element $\varphi \in \operatorname{dom}\left(\tau_{\mu}\right)$ has a (unique) representative for which (6.5) holds for every $x \in[0, b]$; in what follows $\varphi^{\prime}$ will denote this particular representative. From [4, Thm. 2.4] it follows that $\varphi^{\prime}$ may have discontinuities. Indeed,

$$
\varphi^{\prime}(x)-\varphi^{\prime}(x-)=\varphi(x) \mu(\{x\}), \quad x \in(0, b] .
$$

Let $\xi(z, x)$ be the solution to the eigenvalue equation $\tau_{\mu} \varphi=z \varphi, z \in \mathbb{C}$, in the sense given by (6.6), that satisfies the initial conditions $\xi(z, 0)=1, \xi^{[1]}(z, 0)=0$. By [4, Thm. 2.3], this solution exists and is a real entire function of $z$ for every fixed $x \in[0, b]$.

Let $A$ denote the symmetric operator in $L_{2}(0, b)$ given by

$$
\begin{gathered}
\operatorname{dom}(A):=\left\{\begin{array}{c}
\varphi \in \operatorname{dom}\left(\tau_{\mu}\right): \tau_{\mu} \varphi \in L_{2}(0, b), \\
\varphi^{[1]}(0)=\varphi^{\prime}(b-)=\varphi(b)=0
\end{array}\right\}, \\
A \varphi:=-\left(\varphi^{[1]}\right)^{\prime} .
\end{gathered}
$$

The boundary condition $\varphi^{[1]}(0)=0$ is just the usual one $\varphi^{\prime}(0)+h \varphi(0)=0$ with $h=-\mu(\{0\})$. On the other hand, the boundary condition at $b$ is consequence of (6.7) plus the fact that $A$ is the closure of the minimal operator.

Standard arguments yields that $A$ has deficiency indices $(1,1)$ and $\xi(z, \cdot) \in$ $\operatorname{ker}\left(A^{*}-z I\right)$ for all $z \in \mathbb{C}$. Since Green's identity holds true for this kind of operators [4, Thm. 2.2], its canonical selfadjoint extensions are defined in the usual way,

$$
\begin{gathered}
\operatorname{dom}\left(A_{\gamma}\right):=\left\{\begin{array}{c}
\varphi \in \operatorname{dom}\left(\tau_{\mu}\right): \tau_{\mu} \varphi \in L_{2}(0, b), \varphi^{[1]}(0)=0, \\
\varphi(b) \cos \gamma+\varphi^{\prime}(b) \sin \gamma=0, \text { fixed } \gamma \in[0, \pi)
\end{array}\right\}, \\
A_{\gamma} \varphi:=-\left(\varphi^{[1]}\right)^{\prime}
\end{gathered}
$$

The de Branges space associated to $A$ is given by

$$
\mathcal{B}_{A}:=\left\{f(z)=\int_{0}^{b} \xi(z, x) \varphi(x) d x: \varphi \in L_{2}(0, b)\right\}, \quad\|f\|_{\mathcal{B}_{A}}:=\|\varphi\|_{L_{2}(0, b)} .
$$

$\mathcal{B}_{A}$ is isometrically equal to $\mathcal{B}\left(e_{b}\right)$, where $e_{b}(z)=\xi(z, b)+i \xi^{\prime}(z, b)$ is an HermiteBiehler function as shown in [4, Prop. 4.1]. Thus, since $A$ is unitarily equivalent to the multiplication operator in $\mathcal{B}_{A}, A \in \mathcal{S}\left(L_{2}(0, b)\right)$. 
The solutions of the generalized moment problem for $\mathcal{B}_{A}$ correspond to the generalized spectral measures of $A$. Let us consider here the extremal solutions of the generalized moment problem. According to Theorem 4.14 and Remark 4.15, any extremal solution is given by

$$
\rho_{\gamma}=\sum_{\lambda \in \operatorname{spec}\left(A_{\gamma}\right)} \frac{\delta_{\lambda}}{\|\xi(\cdot, \lambda)\|_{L_{2}(0, b)}^{2}},
$$

where $A_{\gamma}$ is a selfadjoint extension of $A$. As in the classical Sturm-Liouville theory, the Fourier transform introduced in (6.9) generates an unitary map between $L_{2}(0, b)$ and $L_{2}\left(\mathbb{R}, d \rho_{\gamma}\right)[4$, Sec. 3].

Theorem 6.2. Let $A$ be the generalized Schrödinger operator defined by $\tau_{\mu}$ as in (6.8). Let $A_{\gamma}$ an arbitrarily chosen selfadjoint extension of $A$ and $\rho_{\gamma}$ the corresponding spectral measure given in (6.10). If

$$
\widetilde{\rho}=\rho_{\gamma}+s \delta_{\lambda}
$$

with $\lambda \notin \operatorname{spec}\left(A_{\gamma}\right)$ and $s>0$, then:

(a) There is no signed Borel measure $\nu$ in $[0, b]$ such that $\widetilde{\rho}$ is the spectral measure corresponding to selfadjoint extensions of $\widetilde{A}$ generated by $\tau_{\nu}$.

(b) The set of entire functions

$$
\left\{f(z):=\int_{0}^{b} \cos (\sqrt{z} x) \varphi(x) d x: \varphi \in L_{2}(0, b)\right\}
$$

is a proper subset of $L_{2}(\mathbb{R}, \widetilde{\rho})$.

Proof. According to [4, Thm. 4.4], the set of functions in the de Branges space $\mathcal{B}_{A}$ coincides with the linear set

$$
\left\{f(z)=\int_{0}^{b} \cos (\sqrt{z} x) \varphi(x) d x: \varphi \in L_{2}(0, b)\right\},
$$

for any signed Borel measure supported in $[0, b]$. Then, assertion (a) is a direct consequence of Theorem 5.4 while (b) follows from Theorems 4.12 and 5.3.

Remark 6.3. We note that regular Schrödinger operators are included in Theorem 6.2 as they correspond to signed Borel measures $\mu$ that are absolutely continuous with respect to the Lebesgue measure, i.e.,

$$
d \mu(x)=q(x) d x \quad \text { with } q \in L_{1}(0, b) .
$$

For these cases, assertion (a) of Theorem 6.2 can be also shown using an elementary argument based on the fact that, the eigenvalues of a regular Schrödinger 
operator with separated boundary conditions (Dirichlet case excluded) obey an asymptotic formula of the form

$$
\lambda_{n}=c n^{2}+O(1), \quad n \rightarrow \infty
$$

for some $c>0$. Clearly, the addition of a point to the spectrum amounts to shifting the enumeration by 1, producing an asymptotic formula with an additional non trivial term linear in $n$ not present in (6.12). An analogous reasoning holds for the Dirichlet case. We remark that Theorem 6.2 holds for a wide class of perturbations of the regular Laplacian for which we do not have at our disposal an asymptotic formula of the kind of (6.12) for the spectra of the corresponding selfadjoint extensions.

The example presented here is also related via [35, Sec. 17] to the GelfandLevitan theory for half-line Schrödinger operators. Let us discuss this in detail. In [13] and [35, Sec. 17] the spectral measure of the half-line Schrödinger operator is defined as follows. Suppose in (6.11) that $q \in L_{1, \text { loc }}([0, \infty)$ ) (with fixed boundary conditions at 0 ) and take into account that $\xi(x, z)$ is the same for any right endpoint $b \in \mathbb{R}$. A spectral measure of the half-line Schrödinger operator is a measure $\rho$ such that the Parseval identity

$$
\|\varphi\|_{L_{2}(0, b)}^{2}=\|f\|_{\mathcal{B}_{A}}^{2}=\int_{\mathbb{R}}|f(\lambda)|^{2} d \rho(\lambda)
$$

holds for every $f=\Phi_{A} \varphi \in \bigcup_{b>0} \mathcal{B}_{A}$ (see (6.9) and compare with [13, Eq. 2] and [35, Thm. 3.2]). The classical Weyl theory tells us that a Schrödinger expression has exactly one spectral measure if $q$ is in the limit point case at infinity, otherwise it has an infinite set of spectral measures. We remark that in the limit circle case, the definition of spectral measure given above includes measures which do not correspond to selfadjoint operators in $L_{2}(0, b)$ (see in $[13$, Sec. 8] complementary conditions for a spectral measure to correspond to selfadjoint extensions).

The central result in Gelfand-Levitan theory is a theorem on necessary and sufficient conditions for a measure to be a spectral measure of a half-line Schrödinger operator, that is, the necesssary and sufficient conditions on $\rho$ such that there is a potential function $q \in L_{1, \mathrm{loc}}([0, \infty))$ ensuring that $(6.13)$ holds. Due to the nonlocality of the conditions (see [13, Introduction]), there is a potential such that a point mass perturbation of a spectral measure is the spectral measure of the half-line Schrödinger operator with that potential and same boundary condition at 0 (see [13, Sec. 7 Example 2] and [26, Chap. 2 Sec. 7]).

We emphasize that the last statement does not contradict Theorem 6.2 since the definition of spectral measure in the Gelfand-Levitan theory is different than the one considered in this work. We believe that a connection between these 
different notions of a spectral measure can be established by resorting to the theory of chains of de Branges spaces developed in [48]. This remains to be done.

To conclude this example, let us revisit the assertion of Theorem 6.2(a) at the light of [4, Secs. 5 and 6]. Since now it is relevant to keep track of the signed measure defining the operators, let us denote by $A(\mu)$ the operator given in (6.8). By [4, Thms. 5.2, 6.1 and 6.2], we know that there is a bijective correspondence between the set of signed Borel measures $\mu$ in $[o, b]$, and the set of even functions $v$ on $(-2 b, 2 b)$ of bounded variation such that $v(0)=-\mu(\{0\})$ and the operator $I+K_{v}$ is positive definite, where

$$
\left(K_{v} \varphi\right)(x)=\frac{1}{2} \int_{0}^{b}(v(t-x)+v(t+x)) \varphi(t) d t
$$

By this correspondence, one has

$$
\|f\|_{\mathcal{B}_{A(\nu)}}^{2}=\left\langle\Phi_{A(0)}^{-1} f,\left(I+K_{v}\right) \Phi_{A(0)}^{-1} f\right\rangle_{L_{2}(0, b)}
$$

Theorem 6.4. Assume the same hypothesis of Theorem 6.2, then there is a signed Borel measure $\nu$ such that $\widetilde{\rho}$ is a non extremal solution to the generalized moment problem for $\mathcal{B}_{A(\nu)}$.

Proof. In view of Theorem 6.2(a), it only remains to prove the existence of a signed Borel measure $\nu$ such that, for the corresponding space $\mathcal{B}_{A(\nu)}$, the equality

$$
\|f\|_{\mathcal{B}_{A(\nu)}}^{2}=\int_{\mathbb{R}}|f(\lambda)|^{2} d \widetilde{\rho}(\lambda)
$$

holds. From the linearity of $K_{v}$ as a map on the function $v$, we can assume without loss of generality that $\rho_{\gamma}$ is given by (6.10) for a selfadjoint extension of $A(0)$. Thus,

$$
\|f\|_{\mathcal{B}_{A(\nu)}}^{2}=\left\|\Phi_{A(0)}^{-1} f\right\|_{L_{2}(0, b)}^{2}+s|f(\lambda)|^{2}
$$

According to [4, Thm. 6.1] (see above), the proof will be established, once we find an even function $v$ on $(-2 b, 2 b)$ of bounded variation such that

$$
\left\langle\Phi_{A(0)}^{-1} f, K_{v} \Phi_{A(0)}^{-1} f\right\rangle=s|f(\lambda)|^{2} .
$$

However, using the notation introduced in Section 4,

$$
\begin{aligned}
s|f(\lambda)|^{2} & =s\left\langle\Phi_{A(0)}^{-1} f, \xi_{A(0)}(\lambda)\right\rangle\left\langle\xi_{A(0)}(\lambda), \Phi_{A(0)}^{-1} f\right\rangle \\
& =\left\langle\Phi_{A(0)}^{-1} f, s\left\langle\xi_{A(0)}(\lambda), \Phi_{A(0)}^{-1} f\right\rangle \xi_{A(0)}\right\rangle .
\end{aligned}
$$

So $K_{v}$ must be equal to $s\left\langle\xi_{A(0)}(\lambda), \cdot\right\rangle \xi_{A(0)}(\lambda)$. Therefore, using the fact that 
$\xi_{A(0)}(\lambda, \cdot)=\cos (\sqrt{\lambda} \cdot)$, we have

$$
\begin{aligned}
\left(K_{v} \varphi\right)(x) & =s \int_{0}^{b} \xi_{A(0)}(\lambda, t) \xi_{A(0)}(\lambda, x) \varphi(t) d t \\
& =s \int_{0}^{b} \cos (\sqrt{\lambda} t) \cos (\sqrt{\lambda} x) \varphi(t) d t \\
& =\frac{s}{2} \int_{0}^{b}(\cos (\sqrt{\lambda}(t+s))+\cos (\sqrt{\lambda}(t-s))) \varphi(t) d t
\end{aligned}
$$

which yields $v(\cdot)=s \cos (\sqrt{\lambda} \cdot)$.

Note that, since $\nu(\{0\})=v(0)$, the point mass perturbation of a extremal spectral measure of a regular Schrödinger operator is a (non extremal) spectral measure of a Schrödinger operator with measure-valued potential $\nu$ such that $\nu(\{0\}) \neq 0$.

\subsection{Bessel operators}

Given $b \in(0, \infty)$, consider the differential expression

$$
\tau_{q}:=-\frac{d^{2}}{d x^{2}}+\frac{\nu^{2}-1 / 4}{x^{2}}+q(x), \quad x \in(0, b), \quad \nu \in[0, \infty) .
$$

We assume that $q \in L_{1, \text { loc }}(0, b)$ is a real-valued function such that $\widetilde{q} \in L_{1}(0, b)$, where

$$
\widetilde{q}(x):= \begin{cases}x q(x) & \text { if } \nu>0 \\ x(1-\log (x)) q(x) & \text { if } \nu=0 .\end{cases}
$$

As shown in [18, Thm. 2.4], $\tau_{q}$ is regular at $x=b$ whereas at $x=0$ it is in the limit point case if $\nu \geq 1$ or in the limit circle case if $\nu \in[0,1)$. For the later case we assume the additional boundary condition

$$
\lim _{x \rightarrow 0+} x^{\nu-\frac{1}{2}}\left((\nu+1 / 2) \varphi(x)-x \varphi^{\prime}(x)\right)=0 .
$$

The expression (6.14), along with the boundary condition (6.15) when $\nu \in$ $[0,1)$, gives rise to a closed, regular, symmetric operator $A$, whose deficiency indices $(1,1)$ [40, Sect. 4].

The corresponding canonical selfadjoint extensions $A_{\gamma}$ are defined as usual,

$$
\operatorname{dom}\left(A_{\gamma}\right):=\left\{\begin{array}{c}
\varphi \in L^{2}(0, b): \varphi, \varphi^{\prime} \in \mathrm{AC}(0, b], \tau_{q} \varphi \in L^{2}(0, b), \\
\text { boundary condition }(6.15) \text { if } \nu \in[0,1), \\
\varphi(b) \cos \gamma+\varphi^{\prime}(b) \sin \gamma=0, \text { fixed } \gamma \in[0, \pi)
\end{array}\right\},
$$


For every $\gamma \in[0, \pi)$, the spectrum of $A_{\gamma}$, beside of being simple and discrete, has at most a finite number of negative eigenvalues [18, Thm. 2.4].

By [18, Lemma 2.2], the eigenvalue equation $\tau \varphi=z \varphi(z \in \mathbb{C})$ admits a solution $\xi(z, x)$, real entire with respect to $z$, with derivative $\xi^{\prime}(z, x)$ also real entire. Moreover, $\xi(z, x)$ also obeys boundary condition (6.15) whenever $\nu \in(0,1)$. This in turn implies $\xi(z, \cdot) \in \operatorname{ker}\left(A^{*}-z I\right)$ for all $z \in \mathbb{C}$. Hence, the associated de Branges space is

$$
\mathcal{B}_{A}:=\left\{f(z)=\int_{0}^{b} \xi(z, x) \varphi(x) d x: \varphi \in L_{2}(0, s)\right\}, \quad\|F\|_{\mathcal{B}_{A}}=\|\varphi\|_{L_{2}(0, b)} .
$$

Also, the extremal solutions of the corresponding generalized moment problem are given by

$$
\rho_{\gamma}=\sum_{\lambda \in \operatorname{spec}\left(A_{\gamma}\right)} \frac{\delta_{\lambda}}{\|\xi(\cdot, \lambda)\|_{L_{2}(0, b)}^{2}},
$$

where $A_{\gamma}$ is any selfadjoint extension of $A$. We now have the following statement analogous to Theorem 6.2.

Theorem 6.5. Suppose $\nu>0$, and $q \in L_{1, l o c}(0, b)$ such that $\widetilde{q} \in L_{r}(0, b)$ for some $r \in(2, \infty]$. Let $A$ be the Bessel operator defined by $\tau_{q}$ as in (6.8). Let $A_{\gamma}$ an arbitrarily chosen selfadjoint extension of $A$ and $\rho_{\gamma}$ the corresponding spectral measure given in (6.16). If

$$
\widetilde{\rho}=\rho_{\gamma}+s \delta_{\lambda}
$$

with $\lambda \notin \operatorname{spec}\left(A_{\gamma}\right)$ and $s>0$, then:

(a) There is no $p \in L_{1, l o c}(0, b)$ with $\widetilde{p} \in L_{r}(0, b)$ for some $r \in(2, \infty]$ such that $\widetilde{\rho}$ is the spectral measure corresponding to selfadjoint extensions of $\widetilde{A}$ generated by $\tau_{p}$.

(b) The set of entire functions

$$
\left\{f(z):=\sqrt{\frac{\pi}{2}} z^{-\frac{\nu}{2}} \int_{0}^{b} \sqrt{x} J_{\nu}(\sqrt{z} x) \varphi(x) d x: \varphi \in L_{2}(0, b)\right\}
$$

is a proper subset of $L_{2}(\mathbb{R}, \widetilde{\rho})$, where $J_{\nu}$ denotes the Bessel function of the first kind.

Proof. The assertions follow from Theorem 4.12, Theorem 5.3 and Corollary 5.4, this time combined with Theorem 4.2 of [40].

Remark 6.6. The requirement on $\widetilde{q}$ stated in Theorem 6.5 is a technical limitation related to the perturbative argument used in the proof of [40, Thm. 4.2]. The technique used there can be easily modified to include $r=2$ but breaks down for $r \in[1,2)$. 
We emphasize that our theory does not rule out the possibility that $\tilde{\rho}$ is the spectral measure of a Bessel operator for some different value of the parameter $\nu$. In this respect, it is worth noticing the following: Assuming $r \geq 2$ and resorting to [18, Thm. 2.5], one can show that the eigenvalues of $A_{\gamma}$ obeys the asymptotic formula

$$
\lambda_{n}=\frac{\pi^{2}}{b^{2}}\left(n+\kappa_{\nu}\right)^{2}+O\left(n^{1 / 2}\right), \quad n \rightarrow \infty, \quad \kappa_{\nu}:= \begin{cases}\frac{2 \nu+1}{4}, & \gamma \neq 0 \\ \frac{2 \nu-1}{4}, & \gamma=0\end{cases}
$$

This implies that, contrary to the case of regular Schrödinger operators, the addition of a point to the spectrum is still compatible with (6.17) but for a different value of $\nu$, namely, for $\widetilde{\nu}=\nu+2$.

\section{Acknowledgments}

We thank A. Poltoratski for helpful comments which led to Remark 5.6 and G. Teschl for useful remarks. J. H. T. thanks IIMAS-UNAM for their kind hospitality.

\section{References}

[1] N. I. Akhiezer. The classical moment problem and some related questions in analysis. Hafner Publishing Co., New York, 1965.

[2] N. I. Akhiezer and I. M. Glazman. Theory of linear operators in Hilbert space. Dover Publications Inc., New York, 1993.

[3] N. Aronszajn. On a problem of Weyl in the theory of singular SturmLiouville equations. Amer. J. Math., 79:597-610, 1957.

[4] A. Ben Amor and C. Remling. Direct and inverse spectral theory of onedimensional Schrödinger operators with measures. Integr. Equ. Oper. Theory, 52:395-417, 2005.

[5] J. M. Berezans'kil. Expansions in eigenfunctions of selfadjoint operators. Translations of Mathematical Monographs, Vol. 17. American Mathematical Society, Providence, R.I., 1968.

[6] M. S. Birman and M. Z. Solomjak. Spectral theory of selfadjoint operators in Hilbert space. Mathematics and its Applications (Soviet Series). D. Reidel Publishing Co., Dordrecht, 1987. 
[7] D. Cichoń, J. Stochel, and F. H. Szafraniec. Naŭmark extensions for indeterminacy in the moment problem. An example. Indiana Univ. Math. J., 59(6):1947-1970, 2010.

[8] L. de Branges. Some Hilbert spaces of entire functions. Trans. Amer. Math. Soc., 96:259-295, 1960.

[9] L. de Branges. Hilbert spaces of entire functions. Prentice-Hall, Inc., Englewood Cliffs, N.J., 1968.

[10] R. del Rio and L. O. Silva. Stability of determinacy and inverse spectral problems for Jacobi operators. Oper. Matrices, 12(3): 619-641, 2018.

[11] V. Derkach, S. Hassi, M. Malamud, and H. de Snoo. Boundary relations and generalized resolvents of symmetric operators. Russ. J. Math. Phys., 16(1):17-60, 2009.

[12] W. F. Donoghue (Jr.) On the perturbation of spectra, Comm. Pure Appl. Math., 18:559-579, 1965.

[13] I. M. Gelfand and B. M. Levitan. On the determination of a differential equation from its spectral function. Izvestiya Akad. Nauk SSSR. Ser. Mat., 15:309-360, 1951.

[14] F. Gesztesy and G. Teschl. Commutation methods for Jacobi operators. J. Differential Equations. 128(1): 252-299, 1996.

[15] M. L. Gorbachuk and V. I. Gorbachuk. M. G. Krein's lectures on entire operators, volume 97 of Operator Theory: Advances and Applications. Birkhäuser Verlag, Basel, 1997.

[16] S. Hassi and H. de Snoo. One-dimensional graph perturbations of selfadjoint relations. Ann. Acad. Sci. Fenn. Math., 22(1):123-164, 1997.

[17] M. Kaltenbäck and H. Woracek. Pontryagin spaces of entire functions. I. Integral Equations Operator Theory, 33(1):34-97, 1999.

[18] A. Kostenko, A. Sakhnovich and G. Teschl. Inverse eigenvalue problems for perturbed spherical SchrÃüdinger operators. Inverse Probl. 26(10):105013, 14,2010 .

[19] M. Krein. Concerning the resolvents of an Hermitian operator with the deficiency-index $(m, m)$. C. R. (Doklady) Acad. Sci. URSS (N.S.), 52:651$654,1946$. 
[20] M. G. Krein. On a remarkable class of Hermitian operators. C. R. (Doklady) Acad. Sci. URSS (N. S.), 44:175-179, 1944.

[21] M. G. Krein. On Hermitian operators whose deficiency indices are 1. C. R. (Doklady) Acad. Sci. URSS (N. S.), 43:323-326, 1944.

[22] M. G. Krein. On Hermitian operators with deficiency indices equal to one. II. C. R. (Doklady) Acad. Sci. URSS (N. S.), 44:131-134, 1944.

[23] M. G. Krein and G. K. Langer. The defect subspaces and generalized resolvents of a Hermitian operator in the space $\Pi_{\kappa}$. Funkcional. Anal. $i$ Priložen, 5(3):54-69, 1971.

[24] M. G. Krein and G. K. Langer. The defect subspaces and generalized resolvents of a Hermitian operator in the space $\Pi_{\kappa}$. Funkcional. Anal. $i$ Priložen, 5(2):59-71, 1971.

[25] B. Ja. Levin. Distribution of zeros of entire functions, volume 5 of Translations of Mathematical Monographs. American Mathematical Society, Providence, Rhode Island, 1980.

[26] B. M. Levitan. Inverse Sturm-Liouville problems. VSP, Zeist, 1987.

[27] B. M. Levitan and I. S. Sargsjan. Sturm-Liouville and Dirac operators, volume 59 of Mathematics and its Applications (Soviet Series). Kluwer Academic Publishers Group, Dordrecht, 1991.

[28] M. S. Livsič. On an application of the theory of Hermitian operators to the generalized problem of moments. C. R. (Doklady) Acad. Sci. URSS (N.S.), 44:3-7, 1944.

[29] A. Luger, G. Teschl, T. Wöhrer. Asymptotics of the Weyl function for Schrödinger operators with measure-valued potentials. Monatsh. Math. 179: 603-613, 2016.

[30] A. I. Markushevich. Theory of functions of a complex variable. Vol. II. Prentice-Hall, Inc., Englewood Cliffs, N.J., 1965.

[31] M. A. Naümark. Self-adjoint extensions of the second kind of a symmetric operator. Bull. Acad. Sci. URSS. Sér. Math. [Izvestià Akad. Nauk SSSR], 4:53-104, 1940.

[32] M. A. Naumark. Spectral functions of a symmetric operator. Bull. Acad. Sci. URSS. Sér. Math. [Izvestia Akad. Nauk SSSR], 4:277-318, 1940. 
[33] J. Pöschel and E. Trubowitz. Inverse spectral theory, volume 130 of Pure and Applied Mathematics. Academic Press, Inc., Boston, MA, 1987.

[34] M. Reed and B. Simon. Methods of modern mathematical physics. I. Academic Press Inc., New York, second edition, 1980.

[35] C. Remling. Schrödinger operators and de Branges spaces. J. Funct. Anal., 196(2):323-394, 2002.

[36] C. Remling. Spectral theory of canonical systems. De Gruyter, Berlin, Boston 2018.

[37] R. Romanov. Canonical systems and de Branges spaces. Preprint: arXiv: 1408.6022

[38] L. O. Silva and J. H. Toloza. On the spectral characterization of entire operators with deficiency indices (1,1). J. Math. Anal. Appl., 367(2):360$373,2010$.

[39] L. O. Silva and J. H. Toloza. The class of $n$-entire operators. J. Phys. A, 46(2):025202, 23, 2013.

[40] L. O. Silva and J. H. Toloza. A class of $n$-entire Schrödinger operators. Complex Anal. Oper. Theory 8:1581-1599, 2014.

[41] L. O. Silva and J. H. Toloza. De Branges spaces and Kreŭn's theory of entire operators. In Operator theory, pages 549-580. Basel: Springer, 2015.

[42] B. Simon. The classical moment problem as a self-adjoint finite difference operator. Adv. Math., 137(1):82-203, 1998.

[43] A. V. Strauss. Generalized resolvents of symmetric operators. Izvestiya Akad. Nauk SSSR. Ser. Mat., 18:51-86, 1954.

[44] A. V. Strauss. Functional models and generalized spectral functions of symmetric operators. Algebra i Analiz, 10(5):1-76, 1998.

[45] F. H. Szafraniec. Naŭmark dilations and Naŭmark extensions in favour of moment problems. In Operator methods for boundary value problems, volume 404 of London Math. Soc. Lecture Note Ser., pages 275-298. Cambridge Univ. Press, Cambridge, 2012.

[46] G. Teschl. Mathematical methods in quantum mechanics, volume 99 of Graduate Studies in Mathematics. American Mathematical Society, Providence, RI, 2009. With applications to Schrödinger operators. 
[47] J. Weidmann. Linear operators in Hilbert spaces, volume 68 of Graduate Texts in Mathematics. Springer-Verlag, New York, 1980.

[48] H. Woracek Perturbations of chains of de Branges spaces. J. D'Analyse Mathématique 135 271-312, 2018. 